\title{
SEARCHES FOR EXCITED AND NEW LEPTONS AND QUARKS AT THE TEVATRON
}

\author{
H.B. GREENLEE* \\ FOR THE CDF AND DØ COLLABORATIONS \\ Fermilab, Batavia, IL 60510, USA \\ *E-mail: greenlee@fnal.gov
}

\begin{abstract}
This talk summarizes recent results on searches for non-Standard Model fermions at the Tevatron by the CDF and D $\varnothing$ experiments using $p \bar{p}$ collisions with center-of-mass energy $\sqrt{s}=1.96 \mathrm{TeV}$. Results reported here are based on integrated luminosities of about $300-1000 \mathrm{pb}^{-1}$. No evidence for non-Standard Model particles is observed.
\end{abstract}

The Fermilab Tevatron collides protons and antiprotons with center-of-mass energy of $\sqrt{s}=1.96 \mathrm{TeV}$. During the recentlycompleted "Run IIa" data run, the Tevatron experiments CDF and DØ collected data with in integrated luminosity of about $1 \mathrm{fb}^{-1}$. This talk summarizes searches for non-Standard Model based on this dataset, or some portion of it.

\section{Theoretical Overview}

The possible existence of non-Standard Model fermions can generically occur in two ways, namely, as excited fermions (e.g. in compositeness models) or as new fermions.

Excited quarks and leptons have the same flavor and gauge quantum numbers as Standard Model fermions. In addition, they always have additional nonstandard effective interactions due to residual effects of the compositeness binding force. The excited fermion searches reported here are interpreted in terms of the compositeness model of Baur, Spira, and Zerwas (BSZ). ${ }^{1}$ In this model, the novel effective interactions allow excited fermions to be produced singly and to decay singly (in association with an ordinary fermion) via a gauge boson interactions (strength $1 / \Lambda$ ) or a four-fermion contact interactions (strength $4 \pi / \Lambda^{2}$ ), where $\Lambda$ is the compositeness scale. Additional dimension- less coupling constants are generically of order one, and are assumed to be exactly one for the purpose of setting model-dependent limits.

New quarks and leptons have different flavor quantum numbers than known fermions, but typically have standard gauge interactions. New fermions are produced in pairs via standard gauge interactions, and decay via the weak interaction. New fermion searches reported in this talk are interpreted in terms of the model of Bjorken, Pakvasa, and Tuan (BPT). ${ }^{2}$ This model postulates the existence of three additional down-type isosinglet right-handed heavy quarks $(D, S, B)$, that mix with and decay into the corresponding right-handed light quarks, plus $W, Z$, and $H$ bosons.

\section{Excited Muon Search}

Both Tevatron experiments have searched for the associated production of an excited muon and an ordinary muon, $q \bar{q} \rightarrow \mu^{*} \mu$, with the excited muon decaying via decaying via $\mu^{*} \rightarrow$ $\mu \gamma .^{3,4}$ The main Standard Model background is $Z / \gamma \rightarrow \mu \mu$ with a radiated photon (initial or final state radiation).

In addition to requiring two high- $p_{T}$ muons and one high- $p_{T}$ photon, both experiments require a $m_{\mu \gamma}>200 \mathrm{GeV}$. For the CDF analysis, events with $\mu \mu$ invariant 
mass close to $m_{Z}$ are excluded, and the exact $m_{\mu \gamma}$ mass cut depends on the $\mu^{*}$ mass hypothesis. CDF observes 0 events in 371 $\mathrm{pb}^{-1}$ of data with an expected background of $0.37 \pm 0.07$ events. D0 observes 0 events in $380 \mathrm{pb}^{-1}$ of data with an exptected background of $0.17 \pm 0.13$ events.

Under the model assumptions $\Lambda=m_{\mu^{*}}$, dimensionless coupling constants equal to one, and that gauge interactions and contact interactions contribute to both production and decay of $\mu^{*}, \mathrm{CDF}$ and $\mathrm{D} \varnothing$ set a lower $\mu^{*}$ mass limit of $696 \mathrm{GeV}$ and $688 \mathrm{GeV}$ respectively. Excluded regions of the $\Lambda$ vs. $m_{\mu^{*}}$ plane are shown in Fig. 1.
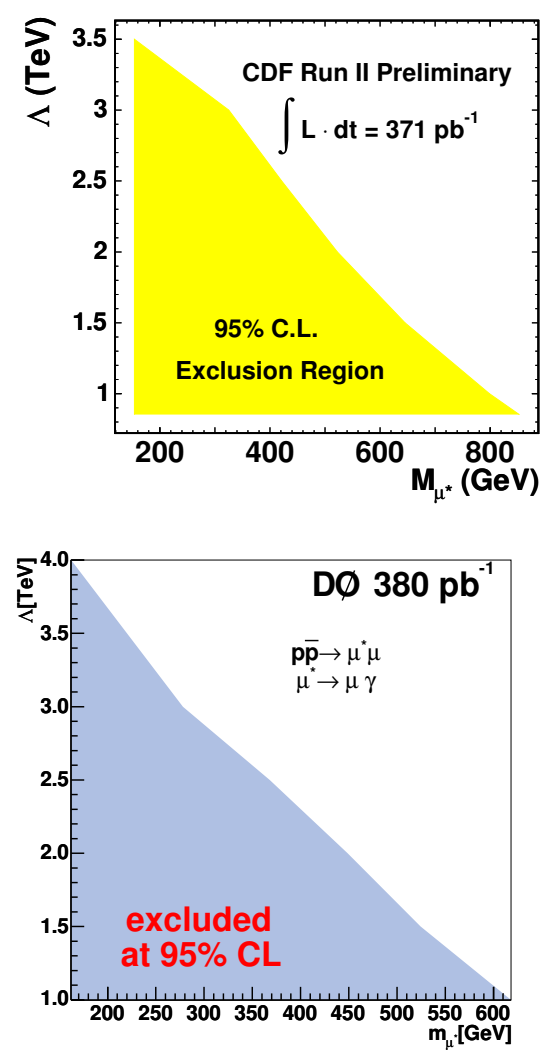

Fig. 1. Excluded region in $\Lambda$ vs. $m_{\mu^{*}}$ for CDF (top) and $\mathrm{D} \varnothing$ (bottom). CDF excluded region assumes that $\mu^{*}$ decays exclusively by gauge interaction.

\section{DØ Excited Quark Search}

The DØ experiment has searched for resonant production of an excited quark via gluon-quark fusion, followed by the decay of the excited quark to $q+Z$, and then $Z \rightarrow e e$. The signature for this reaction is two high$p_{T}$ electrons with invariant mass consistent with $Z$ boson mass, and at least one high$p_{T}$ jet. Furthermore, the jet and the $Z$ boson have fixed invariant mass equal to the $q^{*}$ mass (if the $q^{*}$ is not too wide). Additional selection cuts are used based on $m_{Z \text { jet }}$ and $p_{T Z}$, which are dependent on the $m_{q^{*}}$ and $\Gamma_{q^{*}}$ hypotheses. The main background by far is Standard Model $Z+$ jet production. Figure 2 shows observed events, along with expected background and signal, as a function of $m_{Z \text { jet }}$. The observed events are consistent with background. DØ sets a BSZ model lower limit on the $q^{*}$ mass of $510 \mathrm{GeV}$ assuming $\Lambda=m_{q^{*}}$.

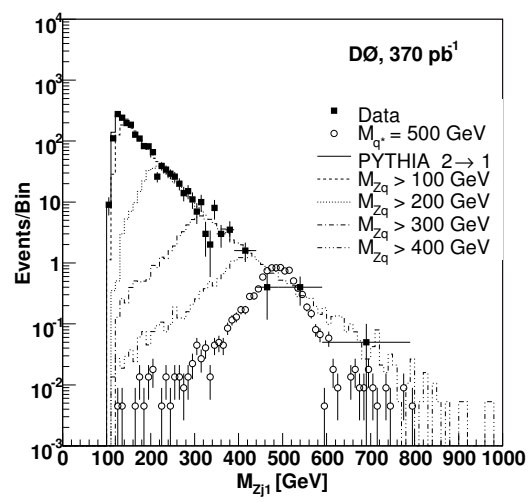

Fig. 2. Observed and expected events vs. $m_{Z \text { jet }}$.

\section{CDF New Quark Searches}

CDF has searched for new quarks that are pair produced by the strong interaction, and that decay into ordinary quarks plus electroweak bosons $W, Z$, or $H$. Obviously, such a process can have many different final states. Rather than looking at a large num- 
ber of different signatures, CDF has concentrated on a few signatures that are promising for discovering non-Standard Model physics (signature-based searches). In this talk, we report results for two such signatures:

- High $p_{T} Z$ 's.

- High mass dileptons $(e e, \mu \mu, e \mu)$.

For concreteness, these searches are interpreted in terms of the BPT model of new quarks described above. ${ }^{2}$

\subsection{High $p_{T} Z$ Search}

For this search, event selection consists of two opposite sign high- $p_{T}$ leptons $(e e$ or $\mu \mu)$ consistent with the $Z$ boson mass, with final selection based on the $p_{T}$ of the lepton pair. The major background is Standard Model $Z+$ jets production. The integrated luminosity is $305 \mathrm{pb}^{-1}$. Figure 3 shows a comparison of observed and expected background events as a function of $p_{T Z}$. No excess above expected background is observed.

This negative result can be expressed in a model-independent fashion as an upper limit on the anomalous $Z$ boson differential cross section $d \sigma / d p_{T Z}$. The result is shown in Fig. 4. In terms of BPT model quarks, CDF sets an upper limit on the production cross section of $\sigma_{Q Q}<0.35 \mathrm{pb}$ for $m_{Q}=300$ $\mathrm{GeV}(95 \% \mathrm{CL})$.

\subsection{High Mass Dilepton Search}

For this search, the basic signature is two high $p_{T}$ isolated leptons ( $e \mu$ or like-sign $e e$, $\mu \mu$ ), plus at least two jets with $E_{T}>50 \mathrm{GeV}$, and $H_{T}>400 \mathrm{GeV}\left(H_{T}\right.$ is the sum of the $E_{T}$ of jets and leptons). The main physics backgrounds are top and diboson production. The integrated luminosity used in this search is $929 \mathrm{pb}^{-1}$. A comparison of data and expected background vs. $H_{T}$ in the $e \mu$ channel, before the jet requirement, is shown in Fig. 5. After the jet requirement, two events remain in data with an expected background
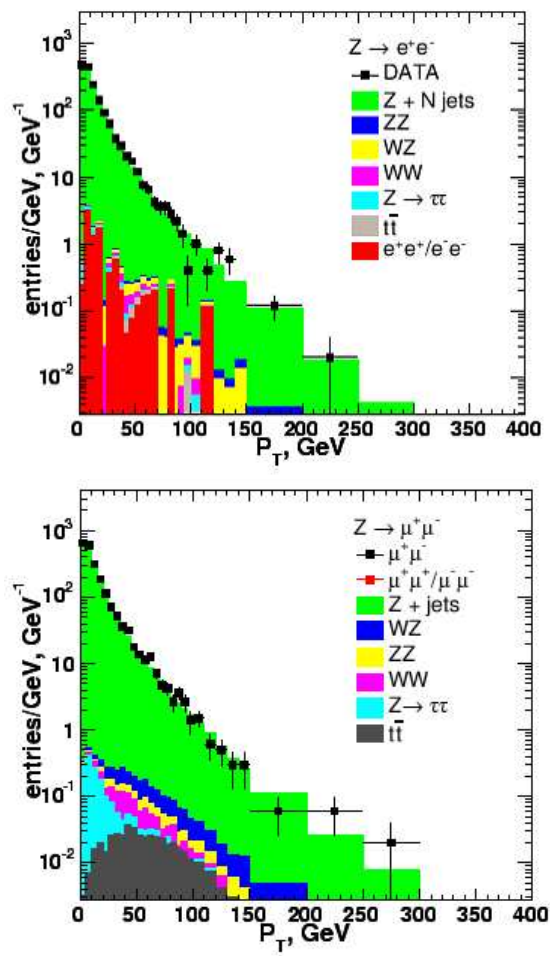

Fig. 3. Observed and expected events vs. $p_{T Z}$ for electrons (top) muons (bottom).

of $1.9 \pm 0.2$ events. For the like-sign $e e$ and $\mu \mu$ channels, no data events pass all cuts, with an expected background of $0.9 \pm 0.09$ events. There is no evidence of non-Standard Model events. This negative result has been used to set an upper limit on the production of 300 $\mathrm{GeV}$ BPT quarks of $\sigma_{Q Q}<0.40 \mathrm{pb}(90 \%$ $\mathrm{CL})$.

\section{Summary and Prospects}

In this talk, results of several searches for non-Standard Model fermions have been presented. No evidence for non-Standard Model physics is seen. Several model-independent and model-dependent limits have been set on nonstandard particles and processes.

Several of the analyses shown here are based on a fraction of the full Run IIa integrated luminosity (about $1 \mathrm{fb}^{-1}$ ). Updated results based on the full Run IIa dataset 

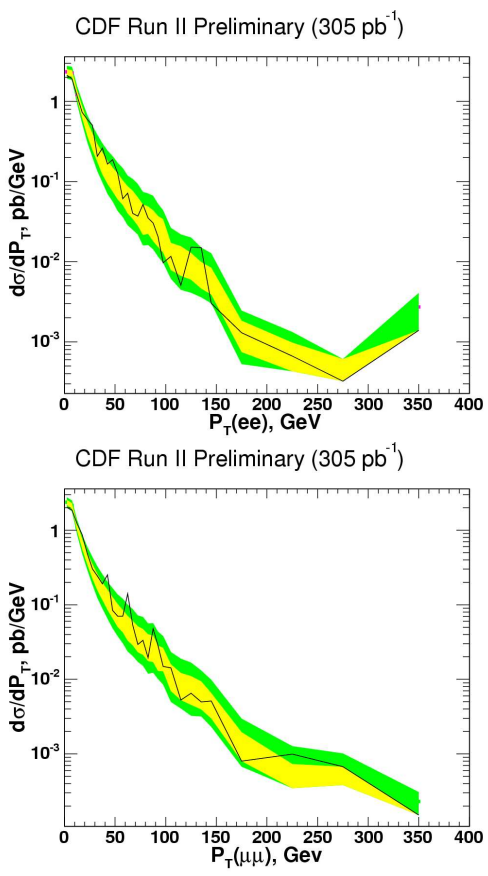

Fig. 4. Upper limit on anomalous $Z$ boson production vs. $p_{T Z}$ for electrons (top) muons (bottom).

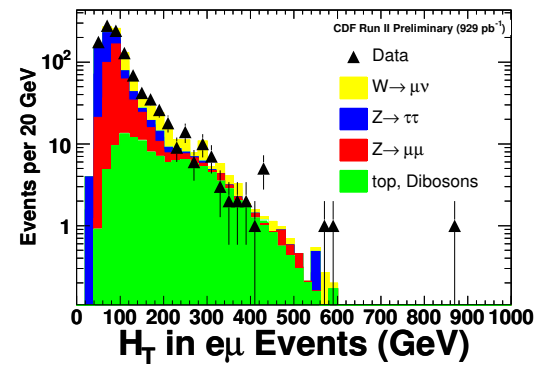

Fig. 5. Observed and expected $e \mu$ events vs. $H_{T}$.

should be forthcoming shortly. The Tevatron experiments are continuing to collect data during the Run IIb data run, which is expected to reach an integrated luminosity of $4-8 \mathrm{fb}^{-1}$ by 2009 .

\section{References}

1. Baur, Spira, and Zerwas, Phys. Rev. D42, 815 (1990).

2. Bjorken, Pakvasa, Tuan, Phys. Rev. D66, 053008 (2002).

3. A. Abulencia et al. CDF Collaboration,
FERMILAB-PUB-06-186-E, submitted to Phys. Rev. Lett.

4. V.M. Abazov et al. (DØ Collaboration), Phys. Rev. D73, 111102 (2006).

5. V.M. Abazov et al. (D $\varnothing$ Collaboration), Phys. Rev. D74, 011104(R) (2006). 